\title{
Multi-Resolution and Multi-Domain Analysis of Off-Road Datasets for Autonomous Driving
}

\author{
Orighomisan Mayuku \\ Mechanical \& Materials Engineering \\ and Ingenuity Labs Research Institute \\ Queen's University \\ Kingston, ON Canada \\ o.mayuku@queensu.ca
}

\author{
Brian W. Surgenor \\ Mechanical \& Materials Engineering \\ and Ingenuity Labs Research Institute \\ Queen's University \\ Kingston, ON Canada \\ surgenor@queensu.ca
}

\author{
Joshua A. Marshall \\ Electrical \& Computer Engineering \\ and Ingenuity Labs Research Institute \\ Queen's University \\ Kingston, ON Canada \\ ORCID 0000-0002-7736-7981
}

\begin{abstract}
For use in off-road autonomous driving applications, we propose and study the use of multi-resolution local binary pattern texture descriptors to improve overall semantic segmentation performance and reduce class imbalance effects in off-road visual datasets. Our experiments, using a challenging publicly available off-road dataset as well as our own off-road dataset, show that texture features provide added flexibility towards reducing class imbalance effects, and that fusing color and texture features can improve segmentation performance. Finally, we demonstrate domain adaptation limitations in nominally similar off-road environments by cross-comparing the segmentation performance of convolutional neural networks trained on both datasets.
\end{abstract}

Index Terms-Features, domain adaptation, segmentation, vision for autonomous vehicles

\section{INTRODUCTION}

Contemporary approaches to autonomous vehicle navigation depend on significant amounts of labelled image data to train convolutional neural networks (CNNs) that are deployed in an end-to-end vision stack. Because CNNs learn by complex representations of shapes, they are well suited to urban environments that have distinct semantic classes, such as cars, person, street light etc. But, in off-road environments, classes are less distinct in shape because of the labelling strategy, which is influenced by the unique challenges encountered in off-road driving. One of these challenges is that, unlike in urban driving, the terrain cannot be taken for granted in off-road driving. Hence, terrain traversability is a key consideration [1]-[4], and in a previous work, we proposed and demonstrated a self-supervised end-to-end method for offroad navigation based on terrain traversal costs [5].

The traversability consideration means that, in off-road datasets, vegetation is often split into non-unique sub-classes, such as grass and traversable grass, based on perceived ease of traversability. Also, in urban driving there is a strict requirement to distinctly identify similar classes-for example, street lights from traffic lights - because the vehicle control strategy will be different in each case. Yet, this is not the case in remote off-road environments. Consequently, in off-road datasets, an

This research work was supported in part by the Natural Sciences and Engineering Research Council of Canada (NSERC) under grant DNDPJ 53339218 and in collaboration with General Dynamics Land Systems (Canada) and Defence Research \& Development Canada (DRDC).

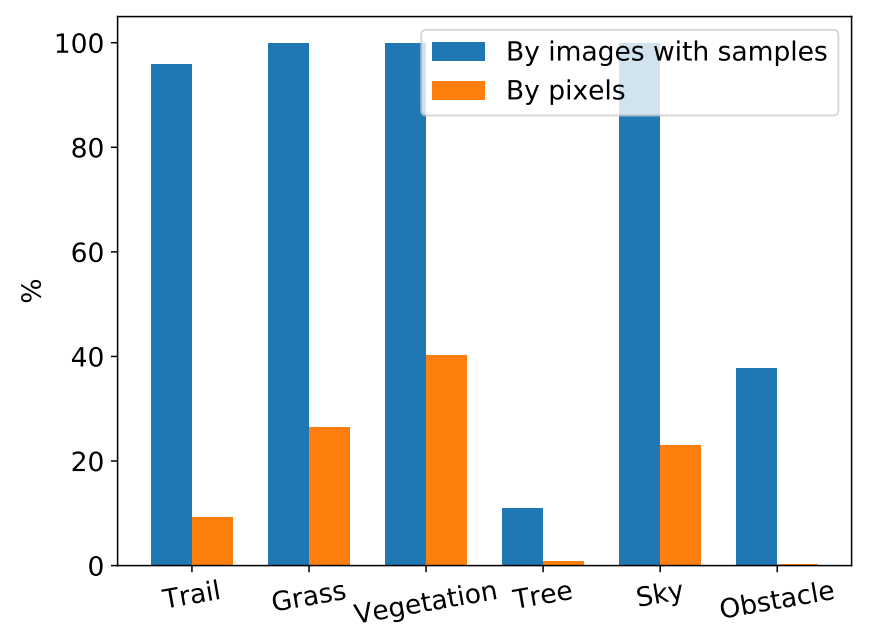

Fig. 1. Imbalance in the Freiburg Forest dataset [6]: the Tree and Obstacle classes are imbalanced; and obstacles appear in more images than trees, but have a smaller pixel count because they are physically smaller, showing spatial imbalance.

obstacle class is often defined comprising an eclectic range of non-unique natural and man-made objects such as rocks of varying sizes and shapes, fences, power pylons, etc. A further complication is the natural class and spatial imbalance in the datasets; e.g., as shown in Fig. 1 for the Freiburg Forest dataset [6] in which obstacles are represented in more images than trees, but occupy fewer pixels showing spatial imbalance.

Whereas classification datasets such as ImageNet can contain millions of images, segmentation datasets contain fewer images because pixel-based labelling entails significant manual effort. What is more, off-road segmentation datasets contain even fewer images because remote off-road environments make data collection less convenient. For example, the Freiburg Forest dataset comprises only 366 images, compared to thousands of images in Cityscapes [7]. The limited size of off-road datasets, combined with the non-unique classes, makes semantic segmentation using $\mathrm{CNNs}$ with RGB-only inputs insufficient.

To specifically tackle the spatial imbalance problem, in 


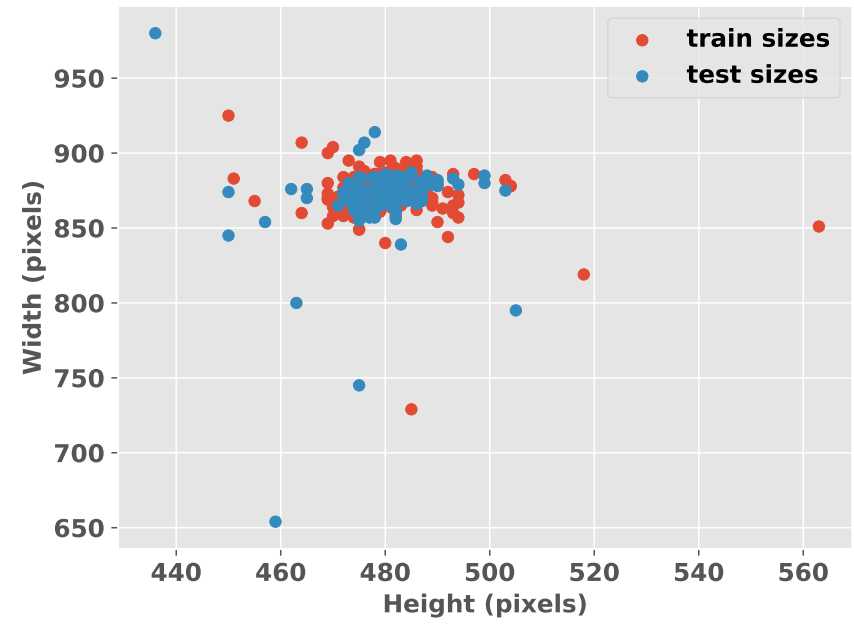

Fig. 2. Image sizes in the Freiburg Forest Dataset. Due to the disparate sizes, the aspect ratios change after resizing to the uniform CNN input size.

addition to augmentation techniques such as zooming, contemporary approaches use deeper networks with flexible convolutions [8], spatial pyramid pooling [9], and dilated convolutions [10] to increase the receptive field of the network in its deeper layers to ameliorate class imbalance effects. But the limited size of the off-road datasets reduces the benefits of these techniques.

One approach to improving segmentation performance is multi-modal fusion, whereby the RGB input is supplemented by other data sources such as infrared and depth data [6]. Apart from the fact that not all of these input modalities are intrinsic properties of the feature classes, e.g. depth, geometric matching of the different sensor inputs is a key issue. Similar pre-processing requirements, such as the need to de-noise stereo disparity maps, limit the scope for online application.

One option for fusion with RGB data that has not yet been explored in this end-to-end context, to the best of our knowledge, is texture. Although CNNs are typically believed to classify objects by shape, they have been shown to have a strong texture bias [11]. While texture has been used in classification tasks in remote sensing [12], [13], image matching [14] and supervised and unsupervised clustering on texture classification benchmarks [15], the texture descriptors have primarily been hand-crafted. Among texture descriptors, the local binary patterns (LBP) descriptor [16] is often preferred due to its ease of computation and robustness [17]. RGB-LBP fusion eliminates the need for geometric matching, which is necessary when using different sensor inputs; and LBP also enables multi-resolution analysis as in [18]. In this work, we study the effectiveness of RGB-LBP fusion.

Finally, off-road environments are less-structured than urban environments due to the natural randomness in the distribution of off-road features; and the considerable effort required to collect, curate and label off-road datasets makes domain adaptability a key concern. There is considerable benefits to be gained if a CNN trained with one dataset can be deployed

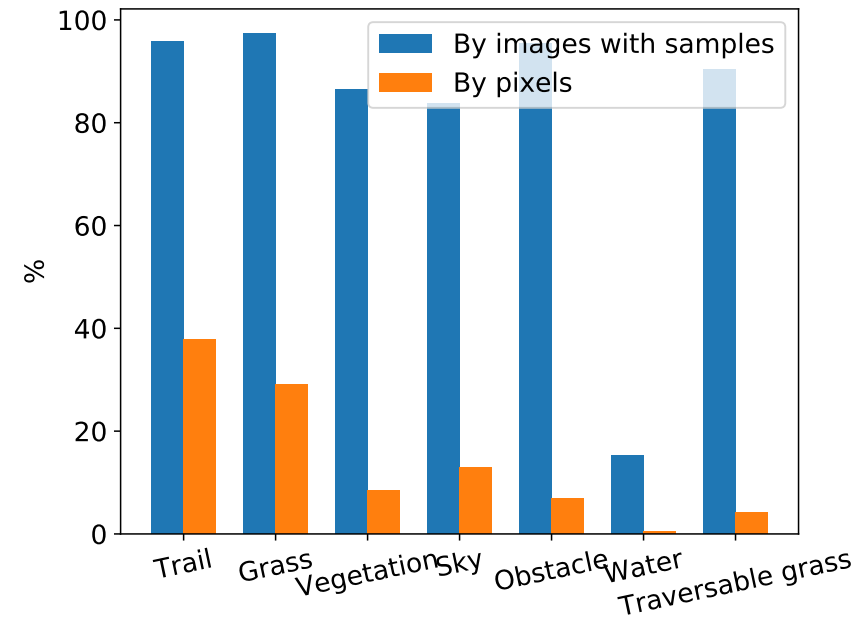

Fig. 3. Imbalance in the SOOR dataset.

in varied off-road environments with little modification.

In the work presented by this paper, we experimentally study the effectiveness of multi-modal fusion of RGB-LBP inputs for improved segmentation performance in challenging offroad datasets. Secondly, we demonstrate domain adaptability limitations in off-road environments by comparing the performance of networks trained on our north American off-road dataset against the Freiburg Forest dataset.

\section{RELATED WORK}

\section{A. Off-Road Datasets and Challenges}

Terrestrial off-road terrains can be forests with trails, or the more diverse Savannah- or prairie-like terrains. Partiallylabelled real-world datasets [19] and synthetic datasets [20] have been used to demonstrate the effectiveness of transfer learning to leverage large classification datasets. But it was also shown that transfer learning is no substitute for a large dataset, and that synthetic datasets must be carefully developed to avoid negative effects in transfer learning.

Although other off-road datasets exist in the literature [21], [22], one predominant publicly available off-road dataset is the Freiburg Forest dataset [6]. However, one critical problem with this dataset is that the images are not of the same size, suggesting inconsistencies in its pre-processing. Aspect ratio is important in CNNs, particularly for smaller features such as those in the Object class. For example, images in benchmark datasets such as Cityscapes [7] are of uniform size. From Fig. 2 , an important question arises: What input size to use in the CNN? In smaller networks [23], a smaller input size not only improves resolution in the encoder, it also mitigates the effects of differing aspect ratios. But deeper networks such as DeepLab [10] require larger and consistent input sizes. In addition, it is unclear why, unlike in the train set, there is no tree sample in the test set. In summary, the dataset is not reasonably uniform enough to allow for full and consistent evaluation. This is one significant motivation for creating our dataset. 
We contend that our own Southern Ontario Off-Road (SOOR) dataset (Fig. 3) is more challenging due to its diversity. In addition to classes in the Freiburg Forest dataset, our SOOR dataset comprises three vegetation subdivisions: Traversable grass, Grass and Vegetation; representative of three levels of difficulty of traversal. It also includes a Water class. The SOOR dataset consists of 257 densely labelled images of size $768 \times 384$ pixels taken on three separate days in July in southern Ontario, Canada. Both datasets exhibit natural class and sample imbalance as shown in Figs. 1 and 3.

\section{B. Multi-Modal Fusion for Improved Scene Understanding}

In [6], multimodal fusion of RGB, depth and near-infrared (NIR) data was studied, and in [21] RGB and LiDAR data fusion was studied. Although fusion with $3 \mathrm{D}$ data can improve segmentation performance, the authors reported that the size of the fused network can limit capacity for deployment online. Also, because of the characteristic noise, stereo disparity maps required refining, and predicting depth with a separate network [24] involves an extra step. It must be noted that, unlike texture, some of these modalities, such as depth and vegetation indices, are not intrinsic properties of all the features in the off-road environment. The later the stage of fusion the more flexible the network is to changes in input modalities. Early or mid-stage fusion is achieved by adding or concatenating the weights or inputs. Late-stage fusion can also be achieved by ensembling or by mixture of experts, which is also applicable to mid-stage fusion. Practically, the most appropriate approach is ascertained by experimentation. A review of multi-modal fusion is presented in [25]. Although late-stage fusion gave the best results in [6], in our work, we use RGB-LBP channel concatenation because it only marginally increases the size of the network and makes for faster inference.

\section{Texture Features}

Texture features include Gabor, Haralick, wavelet, and LBP descriptors; of these, the LBP descriptor is often preferred because of its relative invariance to illumination and rotation [17]. Besides ease of implementation, another advantage of the LBP is its flexibility which has been leveraged for multiresolution analysis [8]. The LBP patterns can be formed in the image plane, thus they fit contemporary end-to-end CNNbased navigation schemes.

\section{Domain Adaptation}

Here, domain adaptation refers to the flexibility to use a CNN, trained on one off-road environment, with images from another off-road environment without labelling. Domain adaptation is commonly treated as a traversability or obstacle avoidance problem [26]-[28] but, practically, in vehicle navigation, obstacle avoidance has long been implemented using 3D sensors [1]. With contemporary semantic segmentation schemes, domain adaptation is the more challenging task of finding correspondences between images at pixel level [29], [30], or as defined in [31]. Here, we experimentally demonstrate the domain adaptation challenge in two off-road environments by cross-comparing CNNs trained on the SOOR and Freiburg Forest datasets.

\section{EXPERIMENTAL APPROACH}

\section{A. The multi-resolution LBP Texture Descriptor}

As shown in Fig. 4, the LBP descriptor uses a binary representation of pixel neighbourhoods to define the texture of image features. For pixel $c$ with intensity $g$ in the grayscale image with a circularly symmetric number of neighbor pixels $P$ within radius $R$, the LBP is defined as [16]

$$
\operatorname{LBP}_{P, R}=\sum_{p=0}^{P-1} 2^{p} s(x),
$$

where

$$
\begin{gathered}
s(x)= \begin{cases}1 & x \geq 0 \\
0 & x<0\end{cases} \\
x=g_{P}-g_{c} .
\end{gathered}
$$

We set $P$ as eight to correspond to the 8-bit images for uniformity during normalization. For multi-resolution analysis, multiple $R$-scale features are combined [18], but we use single $R$ descriptors per fusion. To reasonably capture local information, we limited $R$ to 3, 5 and 7 (see Fig. 4).

\section{B. Implementation, Training and, Augmentation}

Augmentation was done while training using a data generator. Positional augmentation was limited to random vertical and horizontal flips because rotations through acute angles alter labels. Vertical flips improved performance particularly for the irregular shaped water puddles. Colour augmentation was limited to brightening in the range $[0.5, \ldots, 1.5]$. Since brightening affects intensities, it was not done for the labels.

For the Freiburg Forest dataset, we used their validation set for testing. But, during training, their training set was split into the $75-25$ training-validation ratio for early stopping to limit overfitting by monitoring the validation loss profile relative to the training loss profile. And, because the dataset is small, we used three separate training-validation splits to eliminate biases in each validation set. We used the same strategy for our dataset, after keeping $10 \%$ of the dataset for testing. The common learning rate policy was $1-\frac{e}{\max e}$ of the specified initial learning rate, where $e$ is the epoch; with a batch size of four. The learning rate was extensively tuned in each case. To evaluate the techniques on their own merits, we used the basic SGD without regularization methods such as patch extraction or cropping. Our platform was Tensorflow with an NVIDIA Quadro RTX 6000 GPU.

\section{Networks}

We used the FCN8 [23] network architecture as a benchmark with $448 \times 224$ pixels input size, and selected from the Cityscapes leaderboard two notable multi-scale networks, PSPNet and DeepLabv3, that use different multiscale approaches. PSPNet uses spatial pyramid pooling while DeepLabv3 uses dilated convolutions. The implementations 

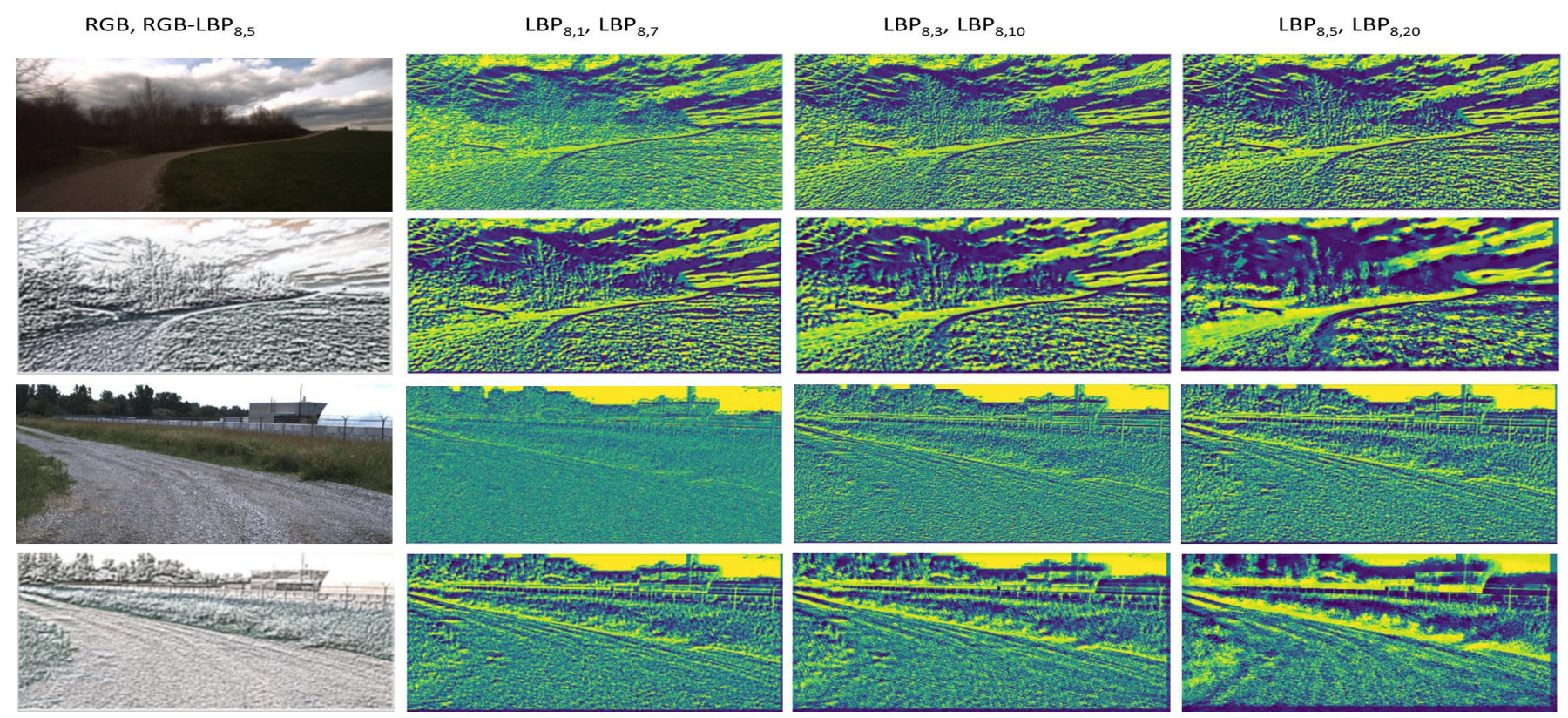

Fig. 4. LBP texture representations for off-road environments at different resolutions. Top two rows, Freiburg Forest dataset sample. Bottom two rows, SOOR dataset sample. First column, upper and lower row pairs are RGB and RGB-LBP (with $R$ value of 5), respectively. From second to fourth column, upper and lower row pair $R$ values are 1 and 7, 3 and 10,5 and 20, respectively. At $R=1$, the per-class texture representations are not visibly distinct because information from the neighbourhood is inadequate. As $R$ increases to 10, the texture patterns become more distinct but the artefacts at the boundaries between classes increase. By $R=20$ the pattern resolution visibly decreases and artefacts increase at the image edges. In [11] it was shown that, in addition to shape, CNNs have a strong texture bias. The internal texture contours provide additional discriminating features for the CNN; and the outer shape for each class is defined by its boundaries in the labelled image.

were based on the authors' papers and their specified repositories. The PSPNet encoder was the same as the FCN8 encoder, but we used a ResNet-50 encoder for DeepLabv3.

\section{RESULTS}

\section{A. Results from FCN8}

Results from the SOOR dataset test set are shown in Table I for two different 75-25 training-validation splits. The metric is the practical intersection over union (IOU) [32] that measures spatial matching between labels and predictions. The difficult classes are the Object, Water and Traversable grass class.

While water puddles in potholes can have similar shapes, they come in different sizes and forms. And, as shown in Fig. 3 , the Water class is the most imbalanced of these, thus it is highlighted in Table I. Water can be uniquely difficult because it reflects other classes in the environment, but this reflection serves to highlight the role of texture in CNNs. As seen in the third figure in Fig. 5, in which the water puddle reflects grass, sky, etc., texture provides added discriminating information to the CNN. The texture network labels a section of the water puddle Sky according to the sky reflection on the water, but the RGB-only network struggles with this distinction. However, the multi-modal RGB-LBP network combines both information for improved segmentation.

Importantly, the significance of texture and shape to CNNs is evident in the texture-only networks in Table I. Two important cases are when $R=1$ and $R=200$. In the former case, the texture features are derived from pixels in the immediate neighbourhood, thus the patterns are not uniquely defined for each class as when $R$ is 3, 5 or 7 (Fig. 4). In the latter case, the texture features are scrambled as they are derived from remote pixels. However, the overall shape of the classes as defined by their respective boundaries in the labelled images provides enough information for reasonable segmentation in the larger classes.

The interactions between traversable grass and the morphologically similar grass are seen in the wrongly segmented sections in Fig. 5. The added texture information does not significantly ameliorate the eclectic nature of the Object class. Hence the relatively poorer segmentation performance in these classes relative to sky and trail. Critically, RGB-texture fusion does not worsen overall segmentation performance.

Results from the Freiburg Forest dataset are shown in Table II. The most difficult class in this dataset is the Objects class (Fig. 1) for the same reasons as in the SOOR dataset. Merging the Tree and Vegetation classes, improves results particularly in the Object class [6]. But we considered the more challenging case by training the CNN with separate Tree and Vegetation classes. However, the Tree class is not represented in their test set, hence the overall performance is not fully evaluated. Although the improvement offered by the multimodal RGB-LBP input over RGB-only input for the Object class is not marked, it is not insignificant. 
TABLE I

FCN8 ON SOOR DATASET (IOU).

\begin{tabular}{|c|c|c|c|c|c|c|c|c|}
\hline \multicolumn{9}{|c|}{ First split. } \\
\hline Modality & $\mathrm{C} 1^{1}$ & $\mathrm{C} 2$ & $\mathrm{C} 3$ & $\mathrm{C} 4$ & $\mathrm{C} 5$ & C6 & C7 & Mean \\
\hline$L B P_{8,1}$ & 0.818 & 0.676 & 0.674 & 0.789 & 0.386 & 0.238 & 0.156 & 0.534 \\
\hline$L B P_{8,3}$ & 0.854 & 0.740 & 0.740 & 0.854 & 0.493 & 0.609 & 0.155 & 0.635 \\
\hline$L B P_{8,5}$ & 0.866 & 0.752 & 0.747 & 0.852 & 0.487 & 0.662 & 0.171 & 0.648 \\
\hline$L B P_{8,7}$ & 0.870 & 0.763 & 0.721 & 0.826 & 0.475 & 0.521 & 0.198 & 0.625 \\
\hline$L B P_{8,10}$ & 0.870 & 0.761 & 0.694 & 0.814 & 0.425 & 0.644 & 0.191 & 0.628 \\
\hline$L B P_{8,200}$ & 0.797 & 0.638 & 0.604 & 0.755 & 0.308 & 0 & 0.016 & 0.445 \\
\hline RGB & 0.908 & 0.817 & 0.806 & 0.914 & 0.609 & 0.617 & 0.350 & 0.717 \\
\hline RGB- $L B P_{8,3}$ & 0.910 & 0.827 & 0.785 & 0.907 & 0.603 & 0.682 & 0.353 & 0.724 \\
\hline RGB- $L B P_{8,5}$ & 0.912 & 0.825 & 0.801 & 0.918 & 0.615 & 0.751 & 0.348 & 0.739 \\
\hline RGB- $L B P_{8,7}$ & 0.912 & 0.830 & 0.802 & 0.909 & 0.619 & 0.754 & 0.357 & 0.741 \\
\hline \multicolumn{9}{|c|}{ Second split. } \\
\hline$L B P_{8,1}$ & 0.809 & 0.647 & 0.680 & 0.814 & 0.330 & 0.016 & 0.130 & 0.489 \\
\hline$L B P_{8,3}$ & 0.854 & 0.739 & 0.728 & 0.835 & 0.438 & 0.543 & 0.190 & 0.618 \\
\hline$L B P_{8,5}$ & 0.869 & 0.753 & 0.725 & 0.835 & 0.451 & 0.653 & 0.247 & 0.648 \\
\hline$L B P_{8,7}$ & 0.865 & 0.758 & 0.741 & 0.849 & 0.481 & 0.600 & 0.195 & 0.641 \\
\hline$L B P_{8,10}$ & 0.875 & 0.758 & 0.732 & 0.851 & 0.436 & 0.595 & 0.185 & 0.633 \\
\hline$L B P_{8,200}$ & 0.836 & 0.647 & 0.612 & 0.827 & 0.356 & 0.093 & 0.069 & 0.492 \\
\hline RGB & 0.903 & 0.806 & 0.786 & 0.904 & 0.562 & 0.609 & 0.296 & 0.695 \\
\hline RGB- $L B P_{8,3}$ & 0.909 & 0.804 & 0.797 & 0.914 & 0.614 & 0.681 & 0.316 & 0.719 \\
\hline RGB- $L B P_{8,5}$ & 0.915 & 0.830 & 0.794 & 0.902 & 0.582 & 0.740 & 0.350 & 0.731 \\
\hline RGB- $L B P_{8,7}$ & 0.910 & 0.811 & 0.796 & 0.905 & 0.615 & 0.703 & 0.326 & 0.724 \\
\hline
\end{tabular}

${ }^{1}$ Class: (1) Trail (2) Grass (3) Vege. (4) Sky (5) Obstacle (6) Water (7) Traversable grass.

RGB
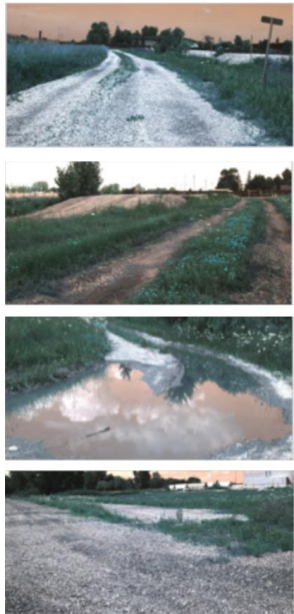

Predicted, RGB
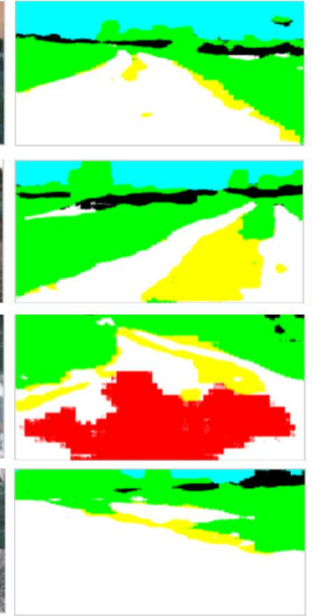

True Label
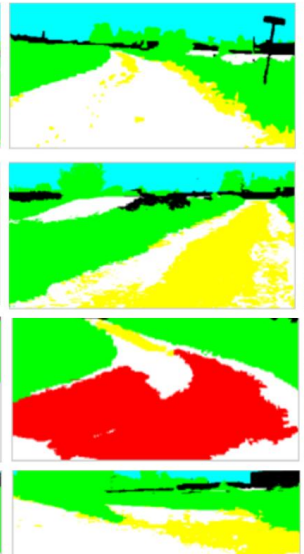

RGB-LBP
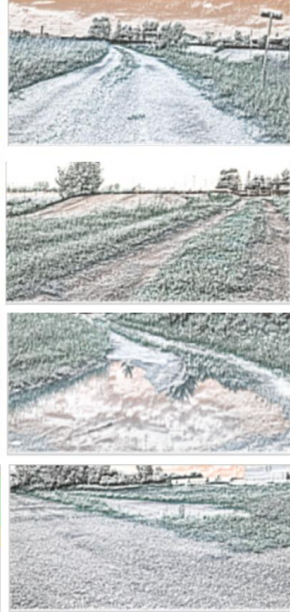

Predicted, RGB-LBP
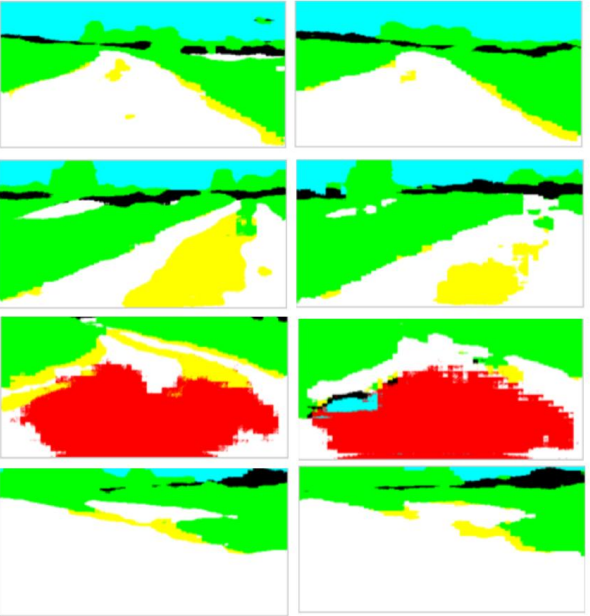

Fig. 5. Predictions from the SOOR dataset. The discretionary basis of distinguishing Traversable grass (yellow) from Grass (green) during labelling means that the $\mathrm{CNN}$ often mislabels them. Also, the texture features provide added information for handling reflections on the water surface in the multi-modal network. $R$ is 5 in all cases. 
TABLE II

FCN8 ON FREIBURG FOREST DATASET (IOU).

\begin{tabular}{ccccccc}
\hline \hline Modality & $\mathrm{C}^{1}$ & $\mathrm{C} 2$ & $\mathrm{C} 3$ & $\mathrm{C} 4$ & $\mathrm{C} 5$ & Mean \\
\hline \hline$L B P_{8,3}$ & 0.692 & 0.766 & 0.841 & 0.879 & 0.157 & 0.556 \\
\hline$L B P_{8,5}$ & 0.709 & 0.703 & 0.848 & 0.875 & 0.148 & 0.559 \\
\hline$L B P_{8,7}$ & 0.720 & 0.782 & 0.840 & 0.873 & 0.166 & 0.564 \\
\hline$L B P_{8,10}$ & 0.701 & 0.767 & 0.840 & 0.866 & 0.099 & 0.545 \\
\hline \hline RGB & 0.846 & 0.857 & 0.881 & 0.910 & 0.200 & 0.616 \\
\hline \hline RGB- $L B P_{8,3}$ & 0.849 & 0.864 & 0.868 & 0.910 & 0.196 & 0.615 \\
\hline RGB- $L B P_{8,5}$ & 0.838 & 0.858 & 0.865 & 0.912 & 0.282 & 0.626 \\
\hline RGB- $L B P_{8,7}$ & 0.850 & 0.862 & 0.874 & 0.907 & 0.274 & 0.623 \\
\hline \hline
\end{tabular}

${ }^{1}$ Class: (1) Trail (2) Grass (3) Vegetation (4) Sky (5) Obstacle.

TABLE III

MULTI-SCALE NETWORKS ON SOOR DATASET (IOU).

\begin{tabular}{c|cc|cc|cc}
\hline & \multicolumn{2}{|c|}{ FCN8 } & \multicolumn{2}{c|}{ PSPNet } & \multicolumn{2}{c}{ DeepLabv3 } \\
\hline \hline Class & In1 $^{1}$ & In2 $^{1}$ & In1 & In2 & In1 & In2 \\
\hline \hline Trail & 0.907 & 0.907 & 0.892 & 0.906 & 0.896 & 0.864 \\
\hline Grass & 0.814 & 0.819 & 0.797 & 0.814 & 0.841 & 0.798 \\
\hline Vegetation & 0.775 & 0.794 & 0.732 & 0.749 & 0.799 & 0.804 \\
\hline Sky & 0.920 & 0.910 & 0.840 & 0.892 & 0.904 & 0.914 \\
\hline Obstacle & 0.573 & 0.601 & 0.541 & 0.536 & 0.591 & 0.599 \\
\hline \hline Water & 0.622 & 0.717 & 0.455 & 0.738 & 0.722 & 0.776 \\
\hline \hline Trav. grass & 0.336 & 0.330 & 0.186 & 0.231 & 0.307 & 0.318 \\
\hline Mean & 0.707 & 0.726 & 0.635 & 0.695 & 0.723 & 0.725 \\
\hline \hline
\end{tabular}

${ }^{1}$ Inputs: (1) RGB (2) RGB- $L B P_{8,5}$

\section{B. Multi-Scale Networks on SOOR Dataset}

The results from the multi-scale networks, shown in Table III, follow the same trend as FCN8. Although, the FCN8 encoder used for PSPNet has limited capacity, and the deeper DeepLabv3 network improves overall performance in the Water class, the results follow the same trend for the Water class as in Table II. The LBP texture features do not interfere with the separate multi-scale techniques used in each network.

\section{Off-Road Domain Adaptability}

Domain adaptation in structured environments, with focus on the domain shift between synthetic and real images, is wellstudied using adversarial learning techniques [33]-[36]. The domain shift between real images of different cities was studied with only one source dataset in [37], [38]. We contend that the domain shift in off-road environments are comparatively larger because of the natural random distribution and variation of environmental features. Also, because the textural patterns of off-road classes are relatively consistent, textural features are relevant [12], [13]. We explore off-road domain adaptation using the Freiburg Forest and SOOR datasets as source and target domains separately, and highlight the effect of texture.

For uniformity, we merged some common classes in each dataset according to Table IV. For the SOOR dataset the Grass and Traversable grass classes were merged; and for the
TABLE IV

DATASET CLASSES FOR MULTI-DOMAIN TESTING.

\begin{tabular}{cc|cc|cc}
\hline \hline \multicolumn{5}{c}{ SOOR Dataset } \\
\hline \hline Trail & Grass & Vege. & Sky & Obstacle & Water \\
\hline \hline \multicolumn{5}{c}{ Freiburg Forest Dataset } \\
\hline \hline Trail & Grass & Vege. & Sky & Obstacle $^{1}$ \\
\hline \hline
\end{tabular}

${ }^{1}$ Does not include water.
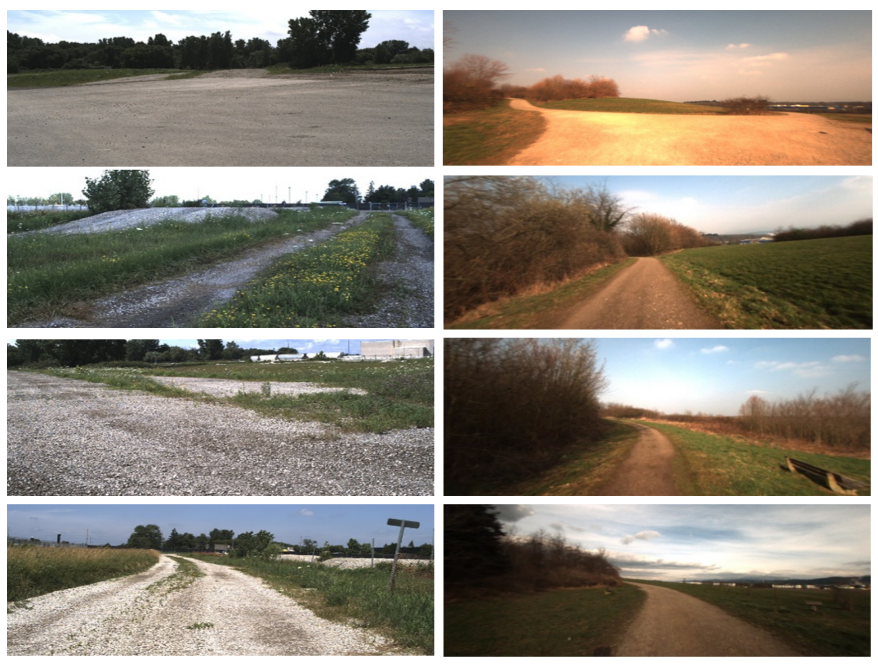

Fig. 6. Sample images from our SOOR (left column) and Freiburg Forest datasets (right column). The images in our SOOR dataset are of higher resolution which aids finer LBP texture representations. Both datasets have grass and dense foliage (Vegetation). However, while the Freiburg Forest dataset has uniform fields of grass, grass in the SOOR dataset is less uniform in height. Similarly, while both Vegetation classes consists of trees, the branches and leaves are denser in the SOOR dataset. In addition, the Trail class in the Freiburg Forest dataset primarily consists of sand, but the SOOR dataset has sand and gravel in its Trail class. Both datasets contain natural and man-made obstacles.

Freiburg Forest dataset, the Tree and Vegetation classes were merged. Images with water were not used in the test. Sample images from each dataset are shown in Fig. 6. In our tests we trained four DeepLabv3 networks on each dataset with RGBonly and RGB-LBP input modalities, respectively.

For the networks trained and evaluated on the same dataset, merging similar classes made each class more distinct and reduced the role of texture as seen in the comparative segmentation performance of both input modalities in Table V. A different fusion strategy [6], [25] or choice of LBP resolution can give different results.

However, the results are different for networks evaluated on a different dataset. As shown in Fig. 6, the Trail, Vegetation and Obstacle classes are the most different across both environments. While networks trained on the higher resolution SOOR dataset gave better results, overall, the best results are for the Sky class, which is the most consistent amongst the classes across both environments. The texturefused networks reflect this consistency, while emphasizing the innate differences in the Grass class, which is less uniform in the SOOR dataset (Fig. 6). 
TABLE V

Multi-Domain Testing Results (IOU).

\begin{tabular}{cccccc}
\hline \hline Input & $\mathrm{C}^{1}$ & $\mathrm{C} 2$ & $\mathrm{C} 3$ & $\mathrm{C} 4$ & $\mathrm{C} 5$ \\
\hline \hline \multicolumn{5}{c}{ Freiburg Forest on Freiburg Forest } \\
\hline \hline In1 ${ }^{2}$ & 0.855 & 0.862 & 0.889 & 0.911 & 0.328 \\
\hline In2 & 0.826 & 0.842 & 0.880 & 0.907 & 0.325 \\
\hline \multicolumn{5}{c}{ Freiburg Forest on SOOR } \\
\hline \hline In1 & 0.007 & 0.220 & 0.100 & 0.289 & 0.001 \\
\hline In2 & 0.074 & 0.048 & 0.121 & 0.460 & 0.000 \\
\hline \hline \multicolumn{5}{c}{ SOOR on S00 } \\
\hline \hline In1 & 0.890 & 0.813 & 0.795 & 0.920 & 0.566 \\
\hline \hline In2 & 0.893 & 0.810 & 0.800 & 0.906 & 0.572 \\
\hline \multicolumn{5}{c}{ SOOR on Freiburg Forest } \\
\hline \hline In1 0.191 & 0.636 & 0.009 & 0.563 & 0.009 \\
\hline In2 0.250 & 0.584 & 0.070 & 0.694 & 0.009 \\
\hline \hline 1 (1) Trail (2) Grass (3) Vege. (4) Sky (5) Obstacle. \\
2 Inputs: (1) RGB (2) RGB- $L B P_{8,7}$ \\
\hline \multicolumn{5}{c}{}
\end{tabular}

\section{CONCLUSION}

We have shown that CNNs are sensitive to LBP texture features at different resolutions, and that fusing RGB and LBP texture features can improve segmentation performance compared to RGB-only segmentation in imbalanced off-road datasets. Like any hyper-parameter, texture parameters, and the fusion strategy, must be tuned by extensive experimentation. Texture features play a role particularly in difficult off-road datasets that have some non-unique classes. Also, we have experimentally demonstrated the domain adaptation problem in off-road environments and shown the importance of texture features across off-road environments.

In order to reduce the tuning effort, we plan to formulate a method for determining the best LBP parameters based on the image parameters. In addition, we hope to experiment with late fusion strategies that make the effect of individual input modalities more pronounced. Finally, we plan to implement a domain adaptation network on both datasets.

\section{REFERENCES}

[1] K. Konolige et al., "Mapping, navigation, and learning for off-road traversal," Journal of Field Robotics, 2009, vol.26, pp. 88-113.

[2] K. Otsu, M. Ono, T. J. Fuchs, I. Baldwin, and T. Kubota, "Autonomous terrain classification with co- and self-training approach," IEEE Robotics and Automation Letters vol. 1, no. 2, pp. 814-819, Jul. 2016.

[3] L. Wellhausen et al., "Where should I walk? Predicting terrain properties from images via self-supervised learning, in IEEE Robotics and Automation Letters, vol. 4, no. 2, pp. 1509-1516, April 2019.

[4] T. Manderson, S. Wapnick, D. Meger, and G. Dudek, "Learning to drive off road on smooth terrain in unstructured environments using an onboard camera and sparse aerial images," in Proceedings of the 2020 IEEE International Conference on Robotics and Automation, June 2020

[5] O. Mayuku, B. W. Surgenor, and J. A. Marshall, "A self-supervised near-to-far approach for terrain-adaptive off-road autonomous driving," to appear in Proceedings of the 2021 IEEE International Conference on Robotics and Automation ICRA, Accepted: February 28, 2021
[6] A. Valada, G. L. Oliveira, T. Brox, and W. Burgard, "Deep multispectral semantic scene understanding of forested environments using multi-modal fusion," in 2016 International Symposium on Experimental Robotics, Nakamura Y., Khatib O., Venture G. Eds, ISER 2016. Springer Proceedings in Advanced Robotics, vol 1. Springer, Cham.

[7] M. Cordts et al., "The cityscapes dataset for semantic urban scene understanding," in Proc. of the IEEE Conference on Computer Vision and Pattern Recognition (CVPR), 2016.

[8] R. Zhang, S. Tang, Y. Zhang, J. Li and S. Yan, "Perspective-adaptive convolutions for scene parsing," in IEEE Transactions on Pattern Analysis and Machine Intelligence, vol. 42, no. 4, pp. 909-924, 1 April 2020

[9] H. Zhao, J. Shi, X. Qi, X. Wang and J. Jia, "Pyramid scene parsing network," in 2017 IEEE Conference on Computer Vision and Pattern Recognition (CVPR), Honolulu, HI, 2017, pp. 6230-6239.

[10] Chen, L.C., Papandreou, G., Schroff, F., Adam, H.: Rethinking atrous convolution for semantic image segmentation, Tech. Report. arXiv:1706.05587 (2017).

[11] R. Geirhos et al., "ImageNet-trained CNNs are biased towards texture; increasing shape bias improves accuracy and robustness," in Proc International Conference on Learning Representations, New Orleans, USA, 2019.

[12] P, Durand, G. Brunet, D. Ghorbanzadeh, and L. Jaupi, "Different approaches for the texture classification of a remote sensing image bank," in Proc. SPIE 10615, Ninth Int. Conf, on Graphic and Image Processing, 1061530, Apr. 2018.

[13] R. Anwer, F. Khan, J. Weijer, M. Molinier, and J. Laaksonen, "Binary patterns encoded convolutional neural networks for texture recognition and remote sensing scene classification," ISPRS Journal of Photogrammetry and Remote Sensing, 2018, vol. 138.

[14] S. Banerji, A. Verma, and C. Liu, "Novel color LBP descriptors for scene and image texture classification," in Proc. 15th Int. Conf. on Image Processing, Computer Vision, and Pattern Recognition, pp. 537-543, July 2011.

[15] A. Porebski, N. Vandenbroucke, and L.Macaire, "Supervised texture classification: color space or texture feature selection?" in Pattern Analysis and Applications, vol. 16, no. 1, pp. 1-18, 2013.

[16] T. Ojala, M. Pietikainen and T. Maenpaa, "Multi-resolution gray-scale and rotation invariant texture classification with local binary patterns," in IEEE Transactions on Pattern Analysis and Machine Intelligence, vol. 24, no. 7, pp. 971-987, July 2002.

[17] L. Liu, P. Fieguth, Y. Guo, X. Wang, M. Pietikäinen "Local binary features for texture classification: Taxonomy and experimental study," in Pattern Recognition, vol. 62, Feb. pp.135-160, 2017.

[18] C.-H. Chan. Multi-scale local binary pattern histogram for face recognition. $\mathrm{PhD}$ thesis, University of Surrey, UK, 2008.

[19] C. J. Holder, T. Breckon, and X. Wei, "From on-road to off: Transfer learning within a deep convolutional neural network for segmentation and classification of off-road scenes," in Proc. European Conference on Computer Vision, 2016, pp. 149-162.

[20] S. Sharma et. al, "Semantic segmentation with transfer learning for off-road autonomous driving," Sensors, vol. 19, no. 11, 2577, 2019.

[21] D.-K. Kim, D. Maturana, M. Uenoyama, and S. Scherer, "Season invariant semantic segmentation with a deep multi-modal network," in Field and Service Robotics, Springer Proceedings in Advanced Robotics, vol. 5, pp. $255-270,2018$.

[22] D. Maturana, P.-W. Chou, M. Uenoyama and S. Scherer, "Real-time semantic mapping for autonomous off-road navigation," in Field and Service Robotics, Springer Proceedings in Advanced Robotics, vol 5. Hutter M., Siegwart R. (eds), Springer, 2018

[23] J. Long, E. Shelhamer and T. Darrell, "Fully convolutional networks for semantic segmentation," in 2015 IEEE Conference on Computer Vision and Pattern Recognition (CVPR), Boston, MA, 2015, pp. 3431-3440.

[24] F. Liu, C. Shen, and G. Lin, "Deep convolutional neural fields for depth estimation from a single image," in Proc. IEEE Conference on Computer Vision and Pattern Recognition (CVPR), Boston, MA, 2015, pp. 51625170.

[25] D. Feng et al., "Deep multi-Modal object detection and semantic segmentation for autonomous driving: Datasets, methods, and challenges," in IEEE Transactions on Intelligent Transportation Systems, '02, 2020, pp. $1-20$.

[26] S. Palazzo et al., "Domain adaptation for outdoor robot traversability estimation from RGB data with safety-preserving Loss," in Proc. of 
the 2020 IEEE/RSJ International Conference on Intelligent Robots and Systems (IROS), Las Vegas, NV, USA, pp. 10014-10021, 2020.

[27] N. Hirose, A. Sadeghian, M. Vázquez, P. Goebel and S. Savarese, "GONet: A semi-supervised deep learning approach for traversability estimation," in 2018 IEEE/RSJ International Conference on Intelligent Robots and Systems (IROS), Madrid, 2018, pp. 3044-3051

[28] G. Cheng, Y. Wang, Y. Qian and J. H. Elder, "Geometry-guided adaptation for road segmentation," in 17th Conference on Computer and Robot Vision (CRV), Ottawa, ON, Canada, 2020, pp. 46-53

[29] Ö. Erkent and C. Laugier, "Semantic segmentation with unsupervised domain adaptation under varying weather conditions for autonomous vehicles," in IEEE Robotics and Automation Letters, vol. 5, no. 2, pp. 3580-3587, April 2020

[30] Y. Zhang, Z. Qiu, T. Yao, D. Liu, and T. Mei, "Fully convolutional adaptation networks for semantic segmentation," in Proc. of the IEEE Conference on Computer Vision and Pattern Recognition (CVPR), June 2018.

[31] J. Hoffman et. al, "CyCADA: Cycle-Consistent adversarial domain adaptation," in Proc. of the International Conference on Machine Learning (ICML), July, 2018, pp. 1994 - 2003,

[32] M. Everingham et al., "The pascal visual object classes challenge: A retrospective," IJCV, vol. 111, iss. 1, 2014

[33] S. Sankaranarayanan, Y. Balaji, A. Jain, S. N. Lim and R. Chellappa, "Learning from synthetic data: Addressing domain shift for semantic segmentation," in Proc. of the 2018 IEEE/CVF Conference on Computer Vision and Pattern Recognition, 2018, pp. 3752-3761

[34] K. Saito, K. Watanabe, Y. Ushiku and T. Harada, "Maximum classifier discrepancy for unsupervised domain adaptation," in Proc. of the 2018 IEEE/CVF Conference on Computer Vision and Pattern Recognition, 2018, pp. 3723-3732

[35] Y. Luo, L. Zheng, T. Guan, J. Yu and Y. Yang, ”Taking a closer look at domain shift: Category-level adversaries for semantics consistent domain adaptation," in Proc. of the 2019 IEEE/CVF Conference on Computer Vision and Pattern Recognition (CVPR), 2019, pp. 2502-2511

[36] Y. Tsai, W. Hung, S. Schulter, K. Sohn, M. Yang and M. Chandraker, "Learning to adapt structured output space for semantic segmentation," in Proc. of the 2018 IEEE/CVF Conference on Computer Vision and Pattern Recognition, 2018, pp. 7472-7481

[37] Y. Zou, Z. Yu, B.V.K. Vijaya Kumar, J. Wang, "Unsupervised domain adaptation for semantic segmentation via class-balanced self-training," in 2018 European Conference on Computer Vision (ECCV) Ferrari V., Hebert M., Sminchisescu C., Weiss Y. Eds. Computer Vision - ECCV 2018. Lecture Notes in Computer Science, vol 11207. Springer, Cham.

[38] Y. Chen, W. Chen, Y. Chen, B. Tsai, Y. F. Wang and M. Sun, "No more discrimination: Cross city adaptation of road scene segmenters," in Proc. of the 2017 IEEE International Conference on Computer Vision (ICCV), 2017, pp. 2011-2020 